\title{
AN APPROXIMATE MATHEMATICAL MODEL FOR SOLIDIFICATION OF A FLOWING LIQUID IN A MICROCHANNEL
}

\author{
T.G. MYERS AND J. LOW
}

\begin{abstract}
A mathematical model is developed to analyse the combined flow and solidification of a liquid in a small pipe or two-dimensional channel. In either case the problem reduces to solving a single equation for the position of the solidification front. Results show that for a large range of flow rates the closure time is approximately constant, and the value depends primarily on the wall temperature and channel width. However, the ice shape at closure will be very different for low and high fluxes. As the flow rate increases the closure time starts to depend on the flow rate until the closure time increases dramatically, subsequently the pipe will never close.
\end{abstract}

\section{INTRODUCTION}

Microfluidic valves are often miniature versions of their larger counterparts. However, due to obvious constraints, such as manufacturing difficulties on a smallscale or lack of space in the microfluidic device, there are also a number of valves that exploit technology not appropriate on a larger scale [5]. In the present paper we discuss one such device, namely the phase change valve.

The phase change valve can work by either displacing a membrane to block the channel or to freeze the fluid within the channel $[5,7,10,17]$. They suffer from an obvious drawback of slow response times, on the order of seconds as opposed to milliseconds for standard valves. However, they do provide a versatile alternative to conventional microvalves when rapid closure is not critical, such as in micro PCR chips [19]. They may also be adapted to a variety of lab on a chip devices $[8,15]$. In high flow rate situations, conventional valves must be robust and consequently are larger and so they generate considerable flow resistance. This is particularly problematic when rows of valves are required. Another issue with standard microvalves is that, in general, they require continuous consumption of energy to remain in an open or closed state [17]. On the other hand, phase change valves are virtually leakproof without utilising much space [8, 19]. Valves that rely on freezing the fluid in the channel have the added advantage that they contain no moving parts [8]. The phase change microvalve developed by Yang and Lin only requires energy during switching of flow status [17].

Key words and phrases. Microvalves; Phase change; Stefan problem; Phase change valve. 
There exist numerous experimental studies into phase change valves. Bevan \& Mutton [2,3] developed a 'freeze-thaw' valve to refine electrophoresis and liquid chromatography processes. He et al [10] developed a phase change valve for a PCR system. This technique was further developed by Chen et al [5] and Gui \& Liu [8]. Chen et al also developed freeze-thaw valves for aqueous and paraffin wax based solutions [5]. The activation time for these devices ranged from 0.5 to 20 seconds.

To date theoretical studies of freezing flow in microchannels have been very limited and in order to make the analysis tractable involve highly restrictive assumptions. For example, Gui \& Liu [8] developed a basic model that assumed plug flow in the liquid, a constant temperature across the pipe, no phase change and a known outlet temperature. Under these assumptions the solution for the fluid temperature is given in the classic text of Carslaw \& Jaeger [4]. However, the result indicated a closing time $t_{c l} \propto 1 / U^{2}$, where $U$ is the average velocity. That is, the faster the fluid moves the more rapid the freezing (a result which the authors recognise as unrealistic). In fact their constant temperature model is consistent with previous models for turbulent flow, where the temperature and velocity are averaged over the cross-section and the boundary condition at the wall then enters as a source term in the governing equation, see [14] for example. In fact, in [14] non-dimensionalisation shows that diffusion along the pipe is negligible in comparison to the advection (by a factor of order $10^{-9}$ !) which considerably simplifies the analysis. The problem with the closure time may then be traced back to incorrectly balancing diffusion with advection. Chen et al [5] study the flow numerically using the FEMLAB package. They also neglect freezing and assume closure occurs instantaneously when the water temperature reaches the value $-17^{\circ} \mathrm{C}$. In different parameter regimes freezing of liquid in pipes is a classical problem, particularly the so-called 'freeze-shut' problem (see the review of [16] for example). Lister \& Dellar [11] study a similar problem in the context of lava solidification. Indeed, our governing equations may be retrieved as a particular limit of theirs.

In the following work we will analyse the flow in a two-dimensional straight sided channel and a circular cross-section pipe subject to a specified wall temperature. A model will be developed for the combined flow and solidification which can then be used to determine the dominant forces driving the freezing and so provide guidelines for the design and operation of a phase change microvalve. In the course of our analysis we find that the freezing time varies with the temperature difference $T_{f}-T_{w}$, where $T_{f}$ and $T_{w}$ are the solidification and wall temperatures respectively. In the numerical calculations we use parameter values for a water-ice system with $T_{f}=273.15 \mathrm{~K}$ (and so neglect supercooling). A number of researchers quote the maximum supercooling as $T_{f} \approx 256.15 \mathrm{~K}$ and use this value in their calculations. However, the actual phase change temperature will depend on the availability of nucleation sites [18] and therefore the smoothness of the channel. Since this will clearly vary widely with the experimental set-up, 
we retain the standard value for water solidification, but stress that the theory is valid for any choice of $T_{f}$. It is also worth pointing out that in the final stages of freezing the phase change temperature will decrease due to the Gibbs-Thompson effect (for example for a water-ice system the melting temperature is reduced by approximately $12 \mathrm{~K}$ when $R=10 \mathrm{~nm}$ ). In common with other studies on freezing in microchannels we will assume this effect to be small and so neglect it.

\section{Mathematical modelling}

2.1. Cartesian freezing. We begin by analysing the two-dimensional Cartesian problem. The configuration is shown in Fig. 1. Fluid flows in the positive $x$-direction due to the action of a pressure gradient. The walls are cooled so that the fluid solidifies. The pipe is assumed to be sufficiently small such that gravity may be neglected.
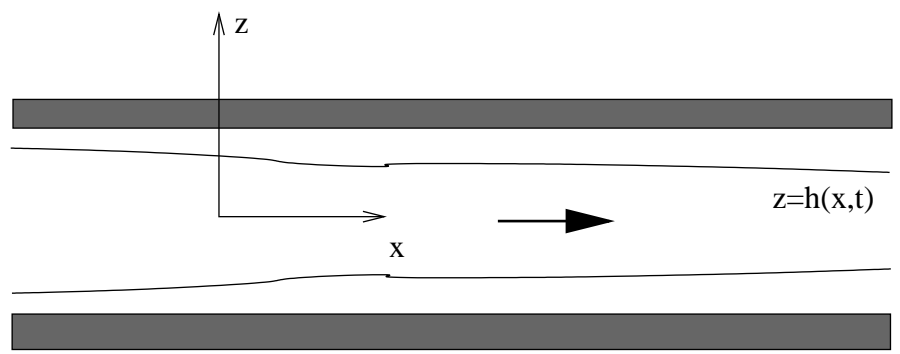

Figure 1. Problem configuration.

In the liquid the flow is governed by the Navier-Stokes equations (1) and the heat equation (2) which includes the advection term. In the solid the heat equation with no advection holds in (3). Finally, the interface between the solid and liquid layers is specified by a Stefan condition (4).

$$
\rho \frac{d \mathbf{u}}{d t}=-\nabla p+\mu \nabla^{2} \mathbf{u}
$$

$$
\begin{array}{cr}
\frac{\partial T}{\partial t}+\mathbf{u} \cdot \nabla T=\alpha_{l} \nabla^{2} T & -h(x, t)<z<h(x, t) \\
\frac{\partial \theta}{\partial t}=\alpha_{s} \nabla^{2} \theta & h(x, t)<|z|<1 \\
\rho_{s} L_{f} \frac{d h}{d t}=\left.k_{s} \frac{\partial \theta}{\partial z}\right|_{z=h}-\left.k_{l} \frac{\partial T}{\partial z}\right|_{z=h} .
\end{array}
$$

In these equations $\mathbf{u}$ is the fluid velocity vector, $T, \theta$ are the temperatures in the solid and liquid layers (and subscripts $s, l$ denote solid and liquid respectively). The constants $\rho, \mu, \alpha, L_{f}$ and $k$ represent density, viscosity, thermal diffusivity, 
latent heat and thermal conductivity, and $\alpha=k /(\rho c)$ where $c$ is the heat capacity. Typical values for ice and water are quoted in Table 1.

\begin{tabular}{||l|l|l||l|l|l||}
\hline \multicolumn{5}{|c|}{ Ice/water } \\
\hline \hline$k_{l}$ & 0.57 & $\mathrm{~W} \mathrm{~m}^{-1} \mathrm{~K}^{-1}$ & $k_{s}$ & 2.18 & $\mathrm{~W} \mathrm{~m}^{-1} \mathrm{~K}^{-1}$ \\
$c_{l}$ & 4181 & $\mathrm{~J} \mathrm{~kg}^{-1} \mathrm{~K}^{-1}$ & $c_{s}$ & 2050 & $\mathrm{~J} \mathrm{~kg}^{-1} \mathrm{~K}^{-1}$ \\
$\rho_{l}$ & 1000 & $\mathrm{~kg} \mathrm{~m}^{-3}$ & $\rho_{s}$ & 917 & $\mathrm{~kg} \mathrm{~m}^{-3}$ \\
$\kappa_{l}$ & $1.35 \times 10^{-7}$ & $\mathrm{~m}^{2} \mathrm{~s}^{-1}$ & $\kappa_{s}$ & $1.16 \times 10^{-6}$ & $\mathrm{~m}^{2} \mathrm{~s}^{-1}$ \\
$L_{f}$ & $3.34 \times 10^{5}$ & $\mathrm{~J} \mathrm{~kg}^{-1}$ & $\mu$ & 0.001 & $\mathrm{~N} \mathrm{~s} \mathrm{~m}^{-2}$ \\
$T_{f}$ & 273.15 & $\mathrm{~K}$ & $T_{0}$ & 283 & $\mathrm{~K}$ \\
$T_{w}$ & 263 & $\mathrm{~K}$ & & & \\
\hline
\end{tabular}

TABLE 1. Typical parameter values for ice and water [13].

In order to sensibly simplify the problem we first non-dimensionalise so that

$$
\begin{aligned}
\bar{x}=\frac{x}{L} & \bar{z}=\frac{z}{R} & \bar{u} & =\frac{u}{U} \quad \bar{w}=\frac{w}{W} \quad \bar{p}=\frac{p}{P} \\
\bar{t} & =\frac{t}{\tau} & \bar{T} & =\frac{T-T_{f}}{T_{o}-T_{f}}
\end{aligned}
$$

where, noting that the geometry is long and thin, the standard lubrication scalings hold and so $W=R U / L, P=\mu U L / R^{2}$. Immediately dropping the overbar notation, this leads to the usual lubrication equations

(7) $\frac{\partial^{2} u}{\partial z^{2}}=\frac{\partial p}{\partial x}+\mathcal{O}\left(\epsilon^{2}, \epsilon^{2} R e\right) \quad \frac{\partial p}{\partial z}=\mathcal{O}\left(\epsilon^{2}, \epsilon^{4} R e\right) \quad \frac{\partial u}{\partial x}+\frac{\partial w}{\partial z}=0$.

Since freezing is the quantity of primary interest, the time-scale $\tau=$ $\rho_{i} L_{f} R^{2} /\left(k_{s}\left(T_{f}-T_{w}\right)\right)$ is taken from the Stefan condition, where we assume freezing is driven through the contact with the wall

$$
\frac{\partial h}{\partial t}=\left.\left(\frac{\partial \theta}{\partial z}-k \frac{\partial T}{\partial z}\right)\right|_{z=h}
$$

where $k=k_{l}\left(T_{o}-T_{f}\right) /\left(k_{s}\left(T_{f}-T_{w}\right)\right)$ is assumed to be $\mathcal{O}(1)$. The heat equation in the solid is

$$
\frac{1}{S} \frac{\partial \theta}{\partial t}=\frac{\partial^{2} \theta}{\partial z^{2}}+\mathcal{O}\left(\epsilon^{2}\right)
$$

where the Stefan number $S=L_{f} /\left(c_{l}\left(T_{f}-T_{w}\right)\right)$. In the liquid we find

$$
\frac{\alpha}{S} \frac{\partial T}{\partial t}+P e\left(u \frac{\partial T}{\partial x}+w \frac{\partial T}{\partial z}\right)=\frac{\partial^{2} T}{\partial z^{2}}+\mathcal{O}\left(\epsilon^{2}\right)
$$

where $\alpha=\alpha_{s}\left(T_{o}-T_{f}\right) /\left(\alpha_{l}\left(T_{f}-T_{w}\right)\right)$ and the Péclet number $P e=U R^{2} /\left(\alpha_{l} L\right)$. 
The flow equations must be solved subject to no-slip at the solid-liquid boundary, symmetry along the centre-line and a mass balance at the solid boundary:

$$
\psi(1, x) \pm h(x, t), t)=0 \quad w(x, 0, t)=0 \quad w(x, h(x, t), t)=\left(1-\frac{\rho_{s}}{\rho_{l}}\right) \frac{L}{U \tau} \frac{\partial h}{\partial t} .
$$

As a first approximation we impose a known, constant temperature at the wall (which is equivalent to specifying a high heat transfer coefficient there). At the phase change boundary the temperature in either phase is the solidification temperature and again there is symmetry at the mid-point:

$$
\left(12 \theta(x, 1, t)=-1 \quad \theta(x, h, t)=T(x, h, t)=\left.0 \quad \frac{\partial T}{\partial z}\right|_{z=0}=0 .\right.
$$

At the inlet the liquid temperature is constant:

$$
T(0, z, t)=1 \text {. }
$$

Note that the dimensional solid temperature is simply denoted $T_{f}$ (and the nondimensional value is -1 ). Although in the results section we set $T_{f}=273.15$ the analysis does not preclude different values for $T_{f}$ to account for supercooling.

Neglecting the obviously small terms, the flow equations may be solved to give

$$
\begin{aligned}
u & =\frac{p_{x}}{2}\left(z^{2}-h^{2}\right) \\
w & =-\frac{\partial}{\partial x} \int_{0}^{z} u(x, \xi, t) d \xi=-\frac{\partial}{\partial x}\left[\frac{p_{x}}{6}\left(z^{3}-3 h^{2} z\right)\right] .
\end{aligned}
$$

An important quantity is the fluid flux

$$
Q=2 \int_{0}^{h} u d z=-\frac{2}{3} h^{3} p_{x} .
$$

Equating the boundary condition for $w(x, h, t)$ in $(11)$ and the value predicted by Eq. (15) gives the mass balance

$$
\frac{L}{U \tau}\left(1-\frac{\rho_{s}}{\rho_{l}}\right) \frac{\partial h}{\partial t}=\frac{\partial}{\partial x}\left[\frac{p_{x} h^{3}}{3}\right] .
$$

This basically states that the liquid layer changes height due to local changes in the flow rate.

Even after the simplifications already made the set of equations is complex, so we now seek further reductions. In the majority of analyses of phase change $\rho_{s}$ is set equal to $\rho_{l}$ which then shows $h^{3} p_{x}=-3 Q / 2$ is independent of $x$. In the present situation this may not be the case, however, provided the product $L /(U \tau)\left(1-\rho_{s} / \rho_{l}\right)$ is small then we may neglect the left hand side of Eq. (17) and thus determine that $Q \approx Q(t)$. Given that $L /(U \tau)$ is the ratio of the flow time-scale to the freezing time-scale it is clear that, except for extremely slow flows (which are of little interest), this ratio is indeed small. If necessary this statement could be verified a priori once the problem has been solved. If we now consider a water-ice system then $S=L_{f} /\left(c_{l}\left(T_{f}-T_{w}\right)\right) \approx 80 /\left(T_{f}-T_{w}\right)$. 
So, for a sensible temperature difference between the wall and the phase change temperature, $S \gg 1$ and the time derivatives are negligible in the heat equations (hence we have what is termed the pseudo-steady approximation, where time drops out of the governing equations but remains present through the influence of the moving boundary). Finally we will work in a co-ordinate system where the flow domain is rectangular $\hat{z} \in[-1,1]$ by using the Landau transformation $\hat{z}=z / h(x, t)$, see $[6, \mathrm{p} 187]$. Then

$$
\begin{gathered}
u=\frac{p_{x} h^{2}}{2}\left(\hat{z}^{2}-1\right)=-\frac{3 Q}{4 h}\left(\hat{z}^{2}-1\right) \\
w(x, \hat{z}, t)=-\frac{\partial}{\partial x}\left[\frac{p_{x} h^{3}}{6}\left(\hat{z}^{3}-3 \hat{z}\right)\right]=\frac{\partial}{\partial x}\left[\frac{Q}{4}\left(\hat{z}^{3}-3 \hat{z}\right)\right] .
\end{gathered}
$$

Noting that $\hat{z}_{x}=-\hat{z} h_{x} / h$ we may express the velocity $w$ as

$$
w(x, \hat{z}, t)=-\frac{3 Q}{4 h} \hat{z} h_{x}\left(\hat{z}^{2}-1\right)=\hat{z} h_{x} u .
$$

The heat flow is described by

$$
P e\left[u\left(\frac{\partial T}{\partial x}-\frac{\hat{z} h_{x}}{h} \frac{\partial T}{\partial \hat{z}}\right)+\frac{w}{h} \frac{\partial T}{\partial \hat{z}}\right]=\frac{1}{h^{2}} \frac{\partial^{2} T}{\partial \hat{z}^{2}}
$$

where we have used $\hat{z}_{t}=-\hat{z} h_{t} / h$. Noting the relation between $u$ and $w$ and substituting for $u$ via Eq. (18) this reduces to

$$
-\frac{3 Q P e}{4}\left(\hat{z}^{2}-1\right) \frac{\partial T}{\partial x}=\frac{1}{h} \frac{\partial^{2} T}{\partial \hat{z}^{2}} .
$$

This simplification is achieved since in the new co-ordinate system the solid boundaries are described by $\hat{z}= \pm 1$ and so there is no 'vertical' flow. The vertical velocity $w$ therefore has no influence in this formulation. An important feature of Eq. (22), given that $h=h(x, t)$, is that it is separable and so may be solved analytically. The thermal boundary conditions are: at $\hat{z}=1 / h, \theta=-1$; at $\hat{z}=1, \theta=T=0$; at $x=0, T=1$; at $\hat{z}=0, T_{z}=0$. By setting $T=X(x) Z(\hat{z})$, Eq. (16) then becomes

$$
-\frac{h(x, t)}{\beta(t)} \frac{X^{\prime}}{X}=\frac{1}{\left(\hat{z}^{2}-1\right)} \frac{Z^{\prime \prime}}{Z}=\lambda^{2}
$$

where $\beta(t)=4 /[3 Q(t) P e]$. This leads to

$$
T=\sum_{n=1}^{\infty} A_{n} e^{-\lambda_{n}^{2} \beta H(x, t)} Z_{n}(\hat{z})
$$

where $H=\int_{0}^{x} 1 / h(\xi, t) d \xi$. The eigenfunctions are

$$
Z_{n}=\frac{W_{m}\left(\lambda_{n} / 4,1 / 4, \lambda_{n} \hat{z}^{2}\right)}{\sqrt{\hat{z}}}+C \frac{W_{w}\left(\lambda_{n} / 4,1 / 4, \lambda_{n} \hat{z}^{2}\right)}{\sqrt{\hat{z}}}
$$


where the constant

$$
C=\frac{\Gamma\left(\left(1-\lambda_{n}\right) / 4\right)}{2 \sqrt{\pi}}=-\left(\frac{3+\lambda_{n}}{8 \sqrt{\pi}}\right) \Gamma\left(-\frac{3+\lambda_{n}}{4}\right)
$$

and $W_{m}, W_{w}$ are the Whittaker $\mathrm{M}$ and $\mathrm{W}$ functions respectively (see [1] for example). The coefficients $A_{n}$ come through orthogonality:

$$
A_{n}=\frac{\int_{0}^{1}\left(1-\hat{z}^{2}\right) Z_{n} d \hat{z}}{\int_{0}^{1}\left(1-\hat{z}^{2}\right) Z_{n}^{2} d \hat{z}} .
$$

The eigenvalues, $\lambda_{n}$, satisfy the transcendental equation

$$
W_{m}\left(\lambda_{n} / 4,1 / 4, \lambda_{n}\right)+C W_{w}\left(\lambda_{n} / 4,1 / 4, \lambda_{n}\right)=0 .
$$

The temperature in the solid may now be written as

$$
\frac{\partial^{2} \theta}{\partial \hat{z}^{2}}=0
$$

hence

$$
\theta=-\frac{h(\hat{z}-1)}{1-h}
$$

Equations (24) and (30) provide explicit expressions for the temperatures in the liquid and solid phases. Substituting for the temperature gradients into the Stefan condition

$$
h \frac{\partial h}{\partial t}=\left.\left(\frac{\partial \theta}{\partial \hat{z}}-k \frac{\partial T}{\partial \hat{z}}\right)\right|_{\hat{z}=1}
$$

results in a single first order integro-differential equation to solve for the position of the freezing front, $h(x, t)$

$$
h \frac{\partial h}{\partial t}=-\frac{h}{1-h}-\left.k \sum_{n=1}^{\infty} A_{n} e^{-\lambda_{n}^{2} \beta(t) H(x, t)} \frac{\partial Z_{n}}{\partial \hat{z}}\right|_{\hat{z}=1}
$$

with initial condition $h(x, 0)=1$. Once $h$ is determined, the pressure, temperature and velocity fields may be determined from the appropriate equations.

To solve Eq. (32) we must first consider the driving force. Mathematically the simplest situation is to specify a constant flux $Q$ (and so $\beta$ is constant) and then allow the pressure to adjust accordingly. However, in reality it is more likely the pressure drop across the channel, $p_{\text {in }}-p_{\text {out }}$, that is specified. Further, fixing $Q$ would prevent us from analysing channel closure, where $Q \rightarrow 0$. In which case we integrate Eq. (16) to determine the time-dependent flux:

$$
Q(t)=\frac{2}{3}\left(p_{\text {in }}-p_{\text {out }}\right)\left[\int_{0}^{1} \frac{1}{h(x, t)^{3}} d x\right]^{-1} .
$$

This specifies $Q(t)$ for a given $\Delta p=p_{\text {in }}-p_{\text {out }}$ and hence $\beta(t)$ which appears in Eq. (32). 
2.2. Freezing in a circular pipe. We now follow a similar analysis for flow through a circular pipe, where $x$ is measured along the pipe axis and $r$ is perpendicular to this. To the same level of approximation as in the previous section the governing equations are

$$
\frac{1}{r} \frac{\partial}{\partial r}\left(r \frac{\partial u}{\partial r}\right)=\frac{\partial p}{\partial x} \quad \frac{\partial p}{\partial r}=0 \quad \frac{\partial u}{\partial x}+\frac{1}{r} \frac{\partial}{\partial r}(r w)=0
$$

where terms of order $\epsilon^{2}$ and $\epsilon^{4} R e$ are neglected. The Stefan condition is unchanged from Eq. (8), with the exception that it is now applied at $r=h(x, t)$. The leading order heat equation in the solid is

$$
\frac{1}{r} \frac{\partial}{\partial r}\left(r \frac{\partial \theta}{\partial r}\right)=0 \text {. }
$$

In the liquid it is

$$
P e\left(u \frac{\partial T}{\partial x}+w \frac{\partial T}{\partial r}\right)=\frac{1}{r} \frac{\partial}{\partial r}\left(r \frac{\partial T}{\partial r}\right) .
$$

The flow equations are subject to

$$
\begin{array}{rrr}
u(x, h(x, t), t)=0 & u_{r}(x, 0, t)=0 & w(x, 0, t)=0 \\
w(x, h(x, t), t) & =\left(1-\frac{\rho_{s}}{\rho_{l}}\right) \frac{L}{U \tau} \frac{\partial h}{\partial t}
\end{array}
$$

and the flux is specified by

$$
Q=2 \pi \int_{0}^{h} r u d r
$$

This leads to

$$
\begin{array}{rlrl}
u & =\frac{p_{x}}{4}\left(r^{2}-h^{2}\right) & Q & =-\frac{\pi}{8} h^{4} p_{x} \\
w & =-\frac{1}{r} \frac{\partial}{\partial x} \int_{0}^{r} \xi u(x, \xi, t) d \xi=-\frac{1}{r} \frac{\partial}{\partial x}\left[\frac{p_{x}}{16} r^{2}\left(r^{2}-2 h^{2}\right)\right] .
\end{array}
$$

Equating the boundary condition for $w(x, h, t)$ and the value predicted by $w(x, z, t)$ gives the mass balance

$$
\frac{L}{U \tau}\left(1-\frac{\rho_{s}}{\rho_{l}}\right) \frac{\partial h}{\partial t}=\frac{1}{16 h} \frac{\partial}{\partial x}\left[p_{x} h^{4}\right] .
$$

So, provided the left hand side is small, we have that $h^{4} p_{x} \approx-8 Q / \pi$ is a function of time and the pressure-flux relation is

$$
p_{\text {out }}-p_{\text {in }}=-\frac{8 Q(t)}{\pi} \int_{0}^{1} \frac{1}{h(x, t)^{4}} d x .
$$

Setting $\hat{r}=r / h(x, t)$ we obtain

$$
u=\frac{2 Q}{\pi h^{2}}\left(1-\hat{r}^{2}\right)
$$




$$
\frac{Q}{2 \pi \hat{r} h} \frac{\partial}{\partial x}\left(\hat{r}^{4}-2 \hat{r}^{2}\right)=-\frac{2 Q}{\pi h^{2}} \hat{r} h_{x}\left(\hat{r}^{2}-1\right)=\hat{r} h_{x} u .
$$

In the Cartesian problem the heat flow in the liquid involves $h(x, t)$. In the axisymmetric problem it does not appear and so the reduction is somewhat simpler:

$$
\frac{2 P e Q(t)}{\pi}\left(1-\hat{r}^{2}\right) \frac{\partial T}{\partial x}=\frac{1}{\hat{r}} \frac{\partial}{\partial \hat{r}}\left(\hat{r} \frac{\partial T}{\partial \hat{r}}\right)
$$

If we set $T=X(x) R(\hat{r})$ then

$$
\frac{X^{\prime}}{\gamma X}=\frac{1}{\hat{r}\left(1-\hat{r}^{2}\right) R} \frac{\partial}{\partial \hat{r}}\left(\hat{r} \frac{\partial R}{\partial \hat{r}}\right)=-\lambda^{2}
$$

where $\gamma(t)=\pi /[2 P e Q(t)]$. The eigenfunctions are

$$
R_{n}=\frac{W_{m}\left(\lambda_{n} / 4,0, \lambda_{n} \hat{r}^{2}\right)}{\hat{r}}
$$

where the transcendental equation to determine the eigenvalues is

$$
W_{m}\left(\lambda_{n} / 4,0, \lambda_{n}\right)=0 \text {. }
$$

Hence

$$
T=\sum_{n=1}^{\infty} A_{n} e^{-\lambda_{n}^{2} \gamma(t) x} R_{n}(\hat{r}) .
$$

The weight function in the Sturm-Liouville problem, defined by Eq. (47), is $\hat{r}\left(1-\hat{r}^{2}\right)$ and so orthogonality requires

$$
\int_{0}^{1} \hat{r}\left(1-\hat{r}^{2}\right) R_{n} R_{m} d \hat{r}=0
$$

for $n \neq m$. The series coefficients are then determined, after imposing the condition $X(0)=0$, as

$$
A_{n}=\frac{\int_{0}^{1} \hat{r}\left(1-\hat{r}^{2}\right) R_{n} d \hat{r}}{\int_{0}^{1} \hat{r}\left(1-\hat{r}^{2}\right) R_{n}^{2} d \hat{r}}
$$

In the solid the temperature is defined by

$$
\theta=\frac{\ln \hat{r}}{\ln h}
$$

which satisfies $\theta=-1$ at $\hat{r}=1 / h$ and $\theta=0$ at $\hat{r}=1$. The Stefan condition is identical to Eq. (31) except it is applied at $\hat{r}=1$. This leads to the governing equation for the interface position

$$
h \frac{\partial h}{\partial t}=\frac{1}{\ln h}-\left.k \sum_{n=1}^{\infty} A_{n} e^{-\lambda_{n}^{2} \gamma(t) x} \frac{d R_{n}}{d \hat{r}}\right|_{\hat{r}=1} .
$$

Again, once this is solved all other quantities follow from the appropriate equations. 
2.3. Limiting behaviour. Equation (32) has an obvious singularity as $t \rightarrow 0$ and so $h \rightarrow 1$. This is a consequence of imposing a (non-dimensional) temperature of -1 at the wall when the initial fluid temperature is 1 . The jump in temperatures leads to an initially infinite temperature gradient and so as $t \rightarrow 0$ the dominant balance in Eq. (32) is

$$
h \frac{\partial h}{\partial t}=-\frac{h}{1-h},
$$

which integrates immediately to give

$$
h=1-\sqrt{2 t}
$$

(after noting $h=1$ at $t=0$ and $h_{t}<0$ ). Since there is no $x$ dependence in the above equation, initially the problem is one-dimensional. This solution is typical for one-dimensional Stefan problems with a fixed temperature boundary condition where $h_{t} \propto t^{-1 / 2}$ (see [12] for example). If Eq. (56) held for all time the pipe would be totally blocked when $t=1 / 2$. This gives us an indication of the time-scale for calculating solutions with flow.

In general we expect the solution to resemble the small time solution, that is the growth rate depends only on time, when the summation term in Eq. (32) is small. This requires either small $k$ or large $\beta H$ (note $H$ is an increasing function of $x$ and $H=0$ at $x=0$ ). Hence, the shape of the freezing front will be approximately constant if

(1) $k \ll 1$, that is the wall temperature is well below freezing, $T_{w} \gg T_{f}$, and so the wall temperature dominates or the incoming water temperature is close to freezing, $T_{0} \approx T_{f}$, and so has little effect on the process.

(2) $\beta H \gg 1: \beta$ large implies a small Péclet number or small flux, both of which are attained with a narrow channel or slow moving fluid; $H$ large requires either $h$ small or that we are sufficiently far down the pipe that the water temperature is close to freezing.

On the other hand, when the summation term is large, i.e. large $k$ or $P e$ or small $x$ then the water temperature has a strong effect and so we expect a noticeable $x$ variation in the solutions. However, since initially $h \approx 1$, the growth must always be independent of $x$ at early times. Further, as the channel closes, $Q \rightarrow 0$ and so $\beta$ increases also $H=\int_{0}^{x} 1 / h d \xi$ increases so in the final stages, the growth will be independent of $x$. For example, in situations with large $P e$ we would expect the solid growth to initially be flat, it will then show an $x$ variation. As the channel closes the growth will again depend on time and so finally the $x$ dependent profile will grow vertically.

In the pipe, in the limit $t \rightarrow 0$ and $h \rightarrow 1$, the freezing is driven by conduction through the solid and then

$$
h \frac{\partial h}{\partial t} \approx \frac{1}{\ln h}
$$


which leads to

$$
h^{2}(1-2 \ln h)=1-4 t .
$$

This has the same small time approximation as the Cartesian problem $h \approx$ $1-\sqrt{2 t}$. Similar comments to the Cartesian case also hold with regards to the behaviour for large and small $k$ and $P e$ and $x$.

\section{NUMERICAL SOLUTION}

In both Cartesian and cylindrical polar co-ordinates the problem reduces to solving the differential equation for $h$, Eq. (32) or (54). We will first describe the solution of the Cartesian case before briefly discussing the simpler radial case.

3.1. The Cartesian model. Equation (32) may be discretised as follows:

$$
\frac{h_{j}^{i+1}-h_{j}^{i}}{[\delta t]_{i}}=-\frac{1}{1-h_{j}^{i}}-\left.\frac{k}{h_{j}^{i}} \sum_{n=1}^{N} A_{n} \exp \left(-\lambda_{n}^{2} \beta^{i} H_{j}^{i}\right) \frac{\partial Z_{n}}{\partial \hat{z}}\right|_{\hat{z}=1}
$$

where

$$
h_{j}^{i+1}=h\left(x_{j}, t_{i+1}\right) \quad \beta^{i}=\frac{4}{3 Q^{i} P e} \quad H_{j}^{i}=\int_{0}^{x_{j}} \frac{1}{h\left(\xi, t_{i}\right)} d \xi .
$$

The flux required to calculate $\beta^{i}$ is

$$
Q^{i}=\frac{2}{3} \Delta p / \int_{0}^{1} \frac{1}{h\left(\xi, t_{i}\right)} d \xi
$$

Up to $n=11$ the constants $A_{n}$ and eigenvalues $\lambda_{n}$ for odd $n$ are

$$
\begin{aligned}
\left(A_{n}, \lambda_{n}\right)= & (-0.8601,1.6816),(0.2700,5.6699),(-0.1646,9.6682), \\
& (0.1196,13.6677),(-0.0945,17.6674),(0.0783,21.6672) .
\end{aligned}
$$

For even $n$ the eigenfunctions $Z_{n}=0$. Hence we only include odd terms in the summation.

Except in the vicinity of $x=0$ (where $H \sim 0$ ) the exponential term in Eq. (59) decays rapidly with increasing $n$. The number of terms, $N$, taken in the summation was therefore decided by considering the steady-state value of $h=h^{*}$ at $x=0$. For example when $\mathrm{k}=1$, we obtain $h^{*}=0.8466$ for $N=9$ and $h^{*}=0.8603$ for $N=11$. That is, we expect that taking $N=9$ rather than $N=11$ will lead to errors of around $2 \%$. Given the number of approximations already made this seems a reasonable level of accuracy. Hence from now on we set $N=9$ and so use only the first 5 non-zero terms in the summation. Note, to verify this, we also carried a limited number of numerical calculations with $N=11$ but found the results indistinguishable from those obtained with $N=9$.

The algorithm then proceeds as follows: 
(1) Set up a mesh grid for $x$ and $t$. To make the code more efficient we allow $\delta t$ to vary. The grid for the time points is obtained by solving Eq. (32) at $x=0$,

$$
h \frac{\partial h}{\partial t}=-\frac{h}{1-h}-\left.k \sum_{n=1}^{N} A_{n} \frac{\partial Z_{n}}{\partial \hat{z}}\right|_{\hat{z}=1}
$$

using MATLAB's ode45 solver. For the $x$ domain, we use a regular mesh spacing of $\Delta x=0.01$ with 101 grid points from $x=0$ to $x=1$.

(2) Calculate the coefficients for the summation $A_{n},\left.\frac{\partial Z_{n}}{\partial \hat{z}}\right|_{\hat{z}=1}$ and $\lambda_{n}$. Since they do not vary through the calculation or when changing the values of $P e$ and $k$, they only need to be computed once and can then be stored for future calculations. The eigenvalues $\lambda_{n}$ are computed by finding the zeros of equation (28) where each $\lambda_{n}$ lies in the interval $[5.1+4(n-2), 6+$ $4(n-2)]$. The Whittaker functions are represented in MATLAB via the Maple software package. The coefficients $A_{n}$ involve integrals of the eigenfunctions, see Eq. (27). Near $z=0$ this leads to numerical difficulties due to the singular behaviour of the components of the eigenfunctions. Consequently, as $\hat{z} \rightarrow 0$ we set

$$
Z_{n}=-\Gamma\left(-\frac{3+\lambda_{n}}{4}\right) \frac{3+\lambda_{n}}{8 \sqrt{\pi}} \frac{\sqrt[4]{\lambda_{n}} \sqrt{\pi}}{\Gamma\left(3 / 4-\lambda_{n} / 4\right)} .
$$

(3) Initial values are specified. Since the governing equation (32) is singular at $t=0$ (when $h=1$ ) we begin calculations at $t_{1}=10^{-6}$, using the small time solution as the initial condition $h(x, t)=1-\sqrt{2 t}$.

(4) The value for the constant pressure drop across the channel is required to calculate the flux $Q(t)$ via Eq. (33). With the flow speed nondimensionalised, we use an initial value of $u=1$ at the channel center $\hat{z}=0$ to obtain an initial flow rate

$$
Q\left(t_{1}\right)=Q^{1}=\frac{4}{3} h_{i n i t}=\frac{4}{3}\left(1-\sqrt{2 t_{1}}\right)
$$

Note, this choice of $u$ determines the constant pressure drop

$$
\Delta p=p_{\text {in }}-p_{\text {out }}=\frac{3 Q^{1}}{2} \int_{0}^{1} \frac{1}{h\left(\xi, t_{1}\right)} d \xi=2 .
$$

The initial value for $\beta$ is

$$
\beta^{1}=\frac{4}{3 Q^{1} \mathrm{Pe}}=\frac{1}{\operatorname{Pe}\left(1-\sqrt{2 t_{1}}\right)} .
$$

(5) The calculation at the next time-step $t_{2}$ is

$$
h_{j}^{2}=h_{j}^{1}-\frac{[\delta t]_{1}}{1-h_{j}^{1}}-\left.\frac{k[\delta t]_{1}}{h_{j}^{1}} \sum_{n=1}^{N} A_{n} \exp \left(-\lambda_{n}^{2} \beta^{1} H_{j}^{1}\right) \frac{\partial Z_{n}}{\partial \hat{z}}\right|_{\hat{z}=1}
$$


for all $j$. At each $j$ node, the integral

$$
H_{j}^{1}=\int_{0}^{x_{j}} \frac{1}{h\left(\xi, t_{1}\right)} d \xi
$$

must be calculated using some quadrature routine. With $h_{2}$ known the new flux value is

$$
Q\left(t_{2}\right)=Q^{2}=\frac{2}{3} \Delta p \int_{0}^{1} \frac{1}{h\left(\xi, t_{2}\right)} d \xi
$$

hence

$$
\beta\left(t_{2}\right)=\beta^{2}=\frac{4}{3 Q\left(t_{2}\right) \mathrm{Pe}} .
$$

The algorithm of steps (68) to (71) may then be repeated until the solution is computed to $t=t_{\text {end }}$. The other variables of interest such as the velocities $u, w$ and the pressure all depend on $h(x, t)$ so they can be calculated once $h$ is known.

For the circular pipe case the computation follows in a similar manner, although it is slightly quicker since there is no need to calculate an integral of the form of Eq. (69) at each node and time-step. The eigenvalues are computed from the zeros of Eq. (50). The first four are $\lambda_{n}=2.70,6.68,10.7,14.7$ which then lead to $A_{n}=0.8978,-0.3119,0.1802,-0.1242$. The constant pressure drop comes from Eq. (43) from an initial flux $Q\left(t_{1}\right)$ using $u=1$ at $\hat{z}=0$ and we calculate $\gamma(t)$ instead of $\beta(t)$. When calculating the coefficients $A_{n}$ for the pipe, we note that the numerator and denominator of $R_{n}(r)$ in Eq. (48), are zero at $r=0$. We avoid the numerical difficulties by applying the formal limit as $r \rightarrow 0$ to obtain $R_{n} \rightarrow \sqrt{\lambda_{n}}$.

\section{Results}

4.1. Evolution of the freezing front. The numerical solutions shown in the following figures use the physical parameter values for water and ice quoted in Table 1. Figure 2 shows the evolution of the ice front for $P e=k=1$ at various non-dimensional times. For small times we see that, except in the vicinity of $x=0$, the front is approximately flat (as predicted by Eq. (55)). As time progresses the ice shape for $x>0.2$ retains the flat shape, whereas for smaller $x$ there is a transition region where the ice shape is curved. The channel is completely blocked when $t \approx 0.5$. Note, this is also the time predicted by the small time solution of $\S 2.3$, by allowing $h \rightarrow 0$. The correspondence is an obvious consequence of the form of governing equation, Eq. (32). If we consider the end point (where closure should first occur) then this is where the exponential term in the summation decays most rapidly. Consequently, provided $\beta$ is not too small, at $x \approx 1 \mathrm{Eq}$. (32) is well approximated by the small time form (55). Figures 3 and 4 show temperature profiles in the water corresponding to times $t=0.0341,0.2379$ of Fig. 2 . The thick solid line denotes the ice-water interface. 


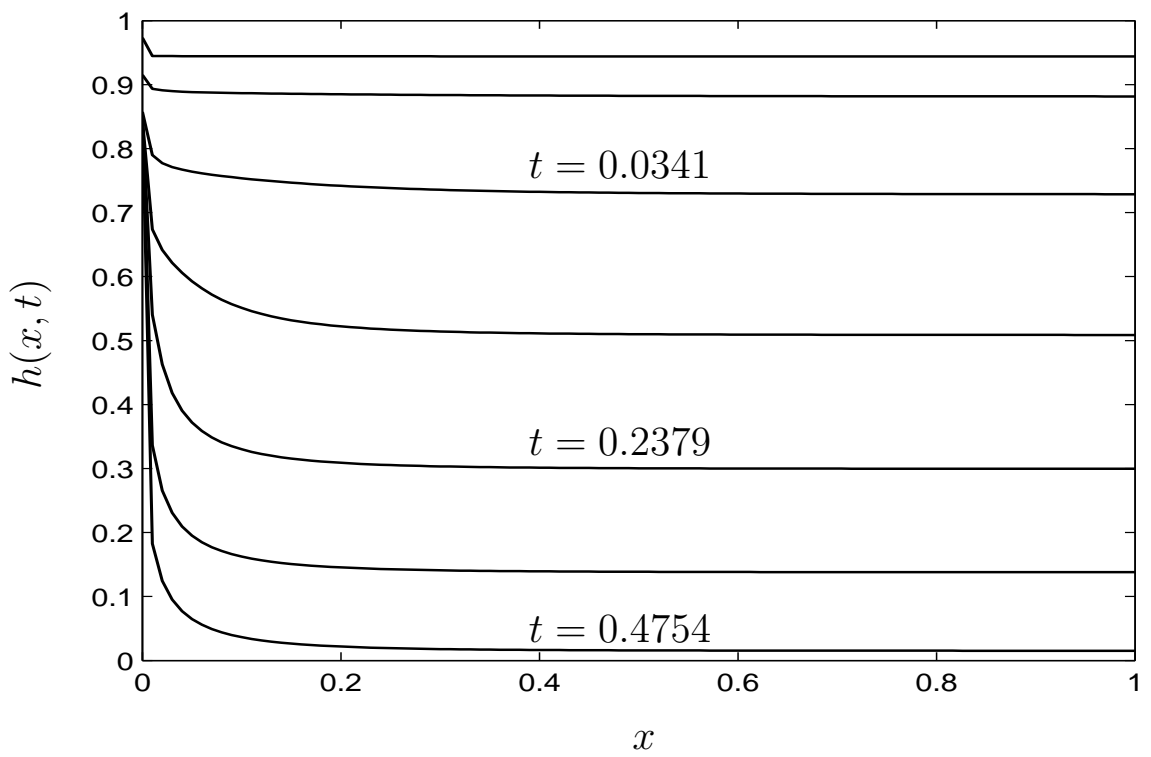

Figure 2. Evolution of the ice front $h(x, t)$ across a Cartesian channel when $\mathrm{Pe}=1$ and $k=1$.

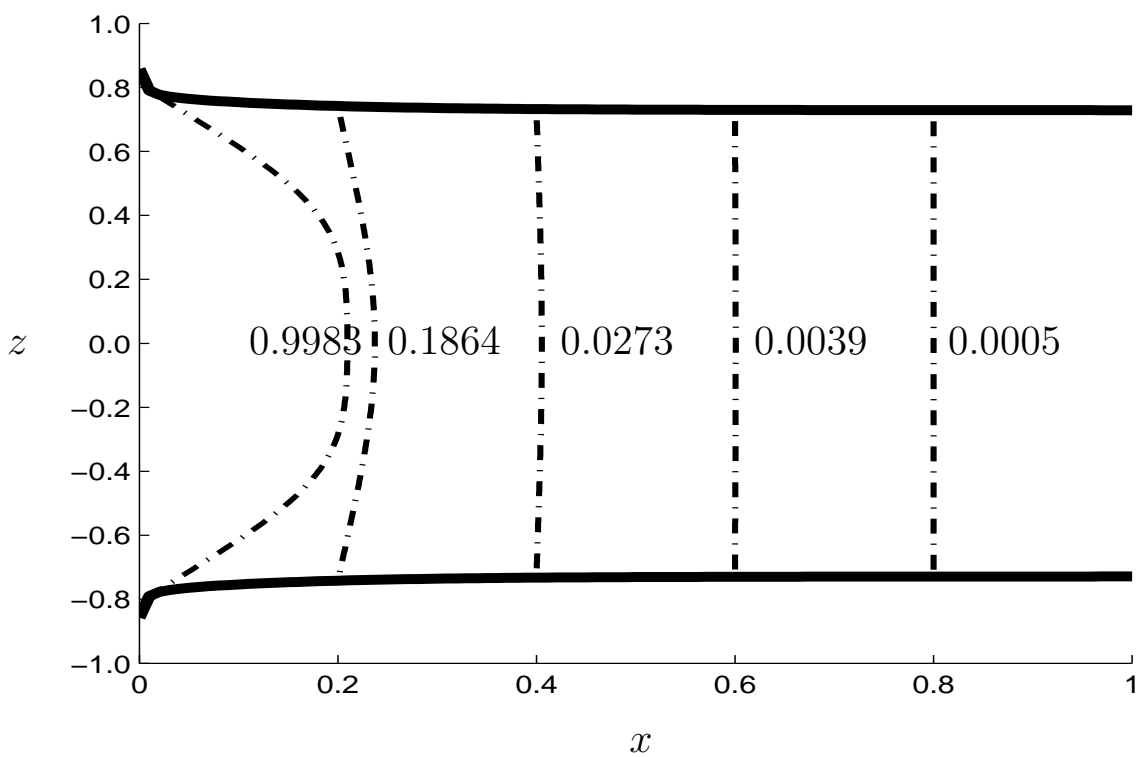

Figure 3. Temperature profiles along the channel corresponding to the ice shapes of Fig. 2 at $t=0.0341$. The ice front is shown as a thick continuous line and the temperature across the channel as a dashed line. 


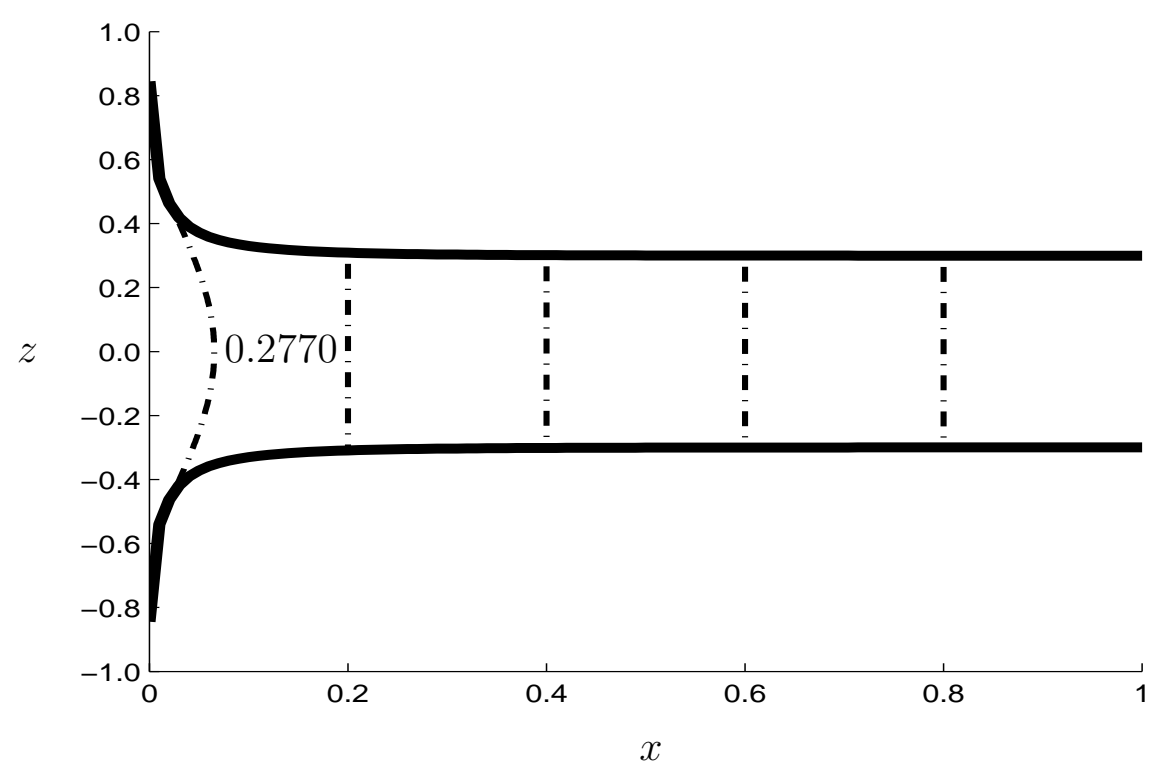

FiguRE 4. Temperature profiles along the channel corresponding to the ice shapes of Fig. 2 at $t=0.2379$. The ice front is shown as a thick continuous line and the temperature across the channel as a dashed line.

The dashed lines denote the water temperature profile measured at the $x$-coordinate where it meets the ice, i.e at $x=0.01,0.2,0.4,0.6,0.8$, at which point $T=T(\hat{r}= \pm 1)=0$. The number associated with each temperature curve is the maximum temperature. So in Fig. 3 we see that liquid entering with temperature $T=1$ rapidly cools at the edges but near the centre $z \in[-0.3,0.3]$ the temperature is close to the initial value. At $x=0.2$ it is significantly cooler with a maximum value $T \approx 0.186$. Moving through the channel the temperature rapidly decreases and by the point $x=0.8$ it is nowhere above $5 \times 10^{-4}$. In Fig. 4 the ice front has moved in, acting to slow the flow, so now the water takes longer to move through the channel and cools earlier. At $x=0.01$ the maximum is $T=0.277$, all subsequent plotted temperatures are below $10^{-4}$ and so the numerical value is not given. A consequence of this is that the temperature gradient in the water is zero and the ice shape is modelled by Eq. (55).

Figure 5 shows the evolution of $h$ when $P e=10$. This is equivalent to increasing the flow rate by a factor 10 . Except for at very small times the ice shape is curved over the whole domain. However, again the closure time is $t \approx 0.5$. This may be interpreted through $\beta=4 /(3 Q P e)$. The decrease in $\beta$ means that the exponential term of Eq. (32) plays an important role over a larger region and so the ice front is no longer flat. However, near $x=1$ it is still sufficiently large that we may approximate the solution with Eq. (55). The value $P e=10$ may be considered a critical value, beyond it the summation term plays an ever 


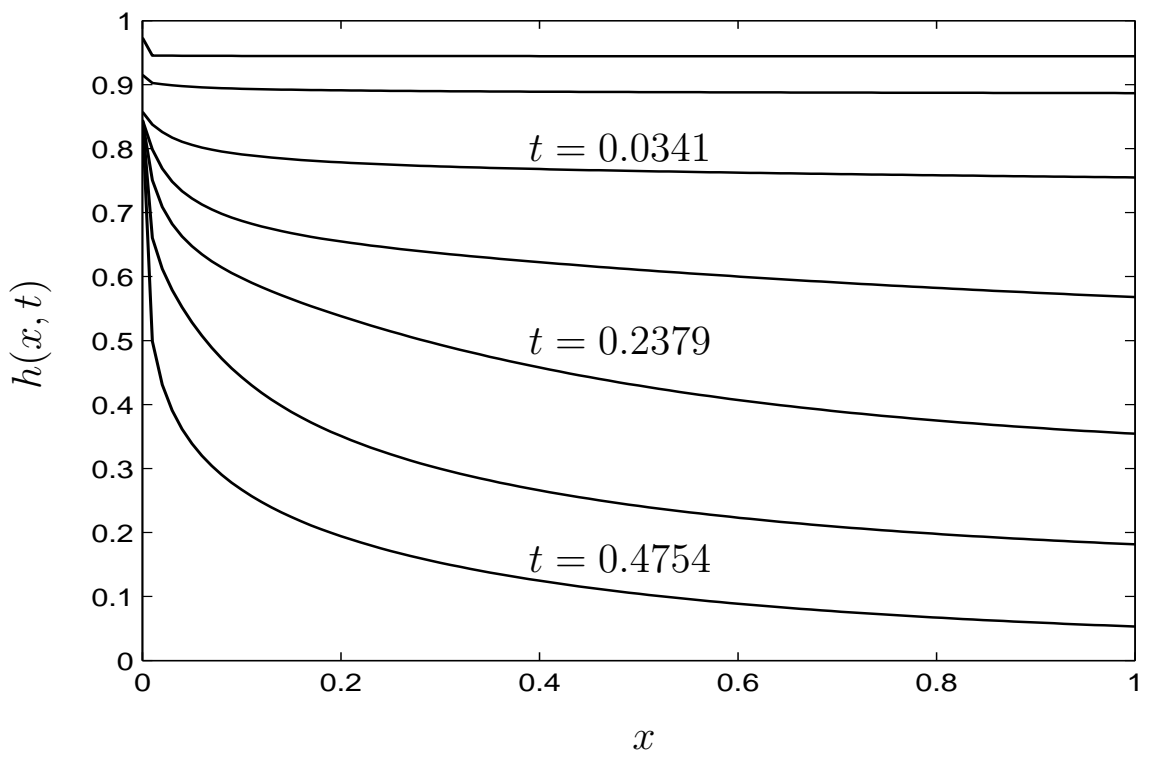

FIGURE 5. Evolution of the ice front $h(x, t)$ across a Cartesian channel when $\mathrm{Pe}=10$ and $k=1$.

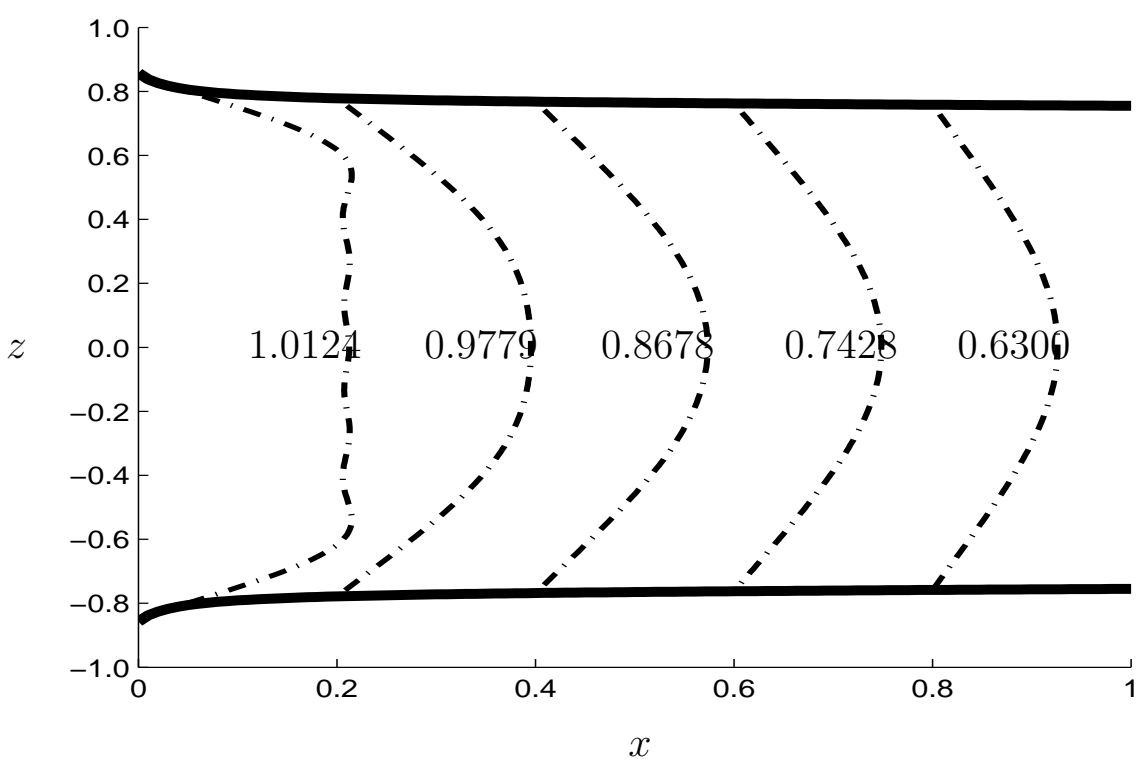

FiguRE 6. Temperature profiles along the channel corresponding to the ice shapes of Fig. 5 at $t=0.0341$. The ice front is shown as a thick continuous line and the temperature across the channel as a dashed line. 


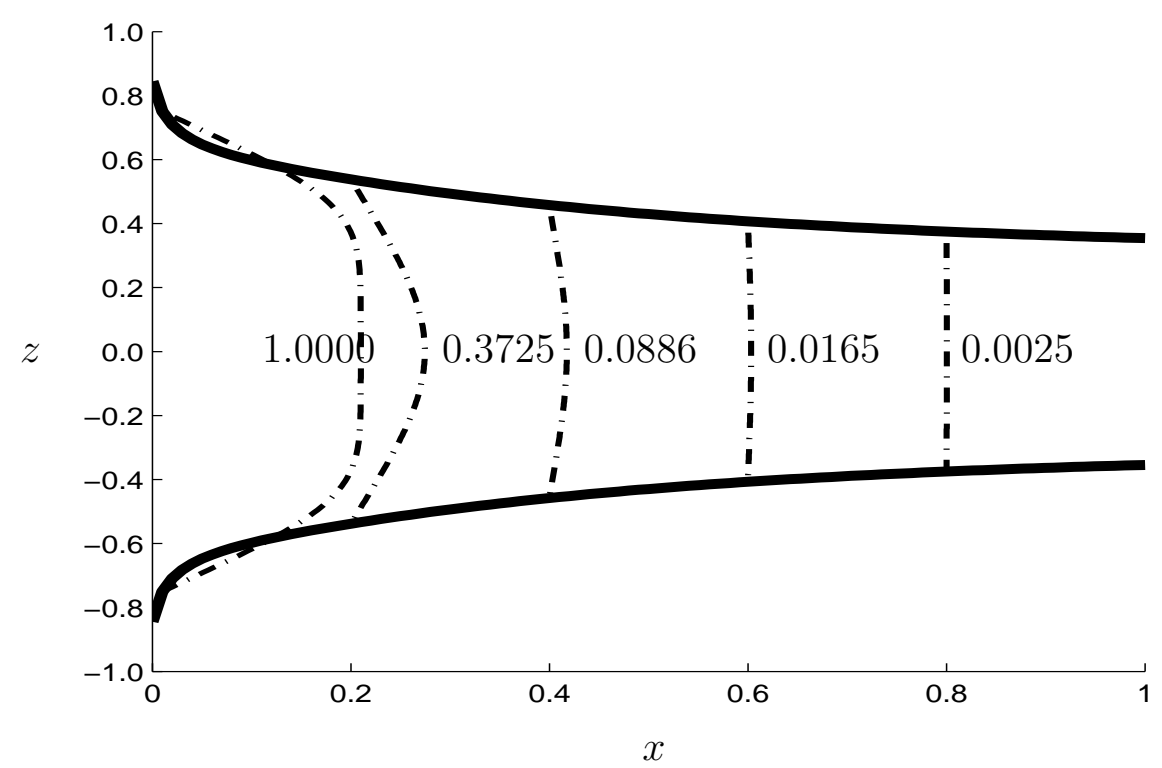

Figure 7 . Temperature profiles along the channel corresponding to the ice shapes of Fig. 5 at $t=0.2379$. The ice front is shown as a thick continuous line and the temperature across the channel as a dashed line.

more significant role and so the closure time starts to increase with $P e$. The behaviour shown in Fig. 5 is exactly as discussed in $\S 2.3$. Initially $h \approx h(t)$ then for $t>0.034$ we see $h=h(x, t)$. For larger times, say $t>0.2379$ the growth for $x$ close to 1 again depends solely on time and so the shape generated at smaller times is retained and moves vertically down on the graph.

Figures 6 and 7 show the temperature in the channel corresponding to the results of Fig. 5 at $t=0.0341,0.2379$. The oscillations on the first temperature curve are a standard problem with Fourier series, known as the Gibbs phenomenon, and results from attempting to model a jump discontinuity $(T=0$ at the ice front but $T=1$ everywhere else) with a combination of continuous functions. This is only an issue in the immediate vicinity of $x=0$ and the oscillations quickly die down. The flux in this case is much greater than that of Figures 3, 4 and so the water can retain more energy as it moves down the channel. At $x=0.8$ the maximum value is $T \approx 0.63$ as opposed to $5 \times 10^{-4}$ on Fig. 3 . The ice shape is approximately flat in this figure since it follows the small time solution, except near $x=0$. However the temperature gradient is clearly non-zero and subsequently the ice must become curved in response to the flow. In Fig. 7 the flux has decreased due to the decrease in channel width and so the temperature also decreases more rapidly with $x$. 


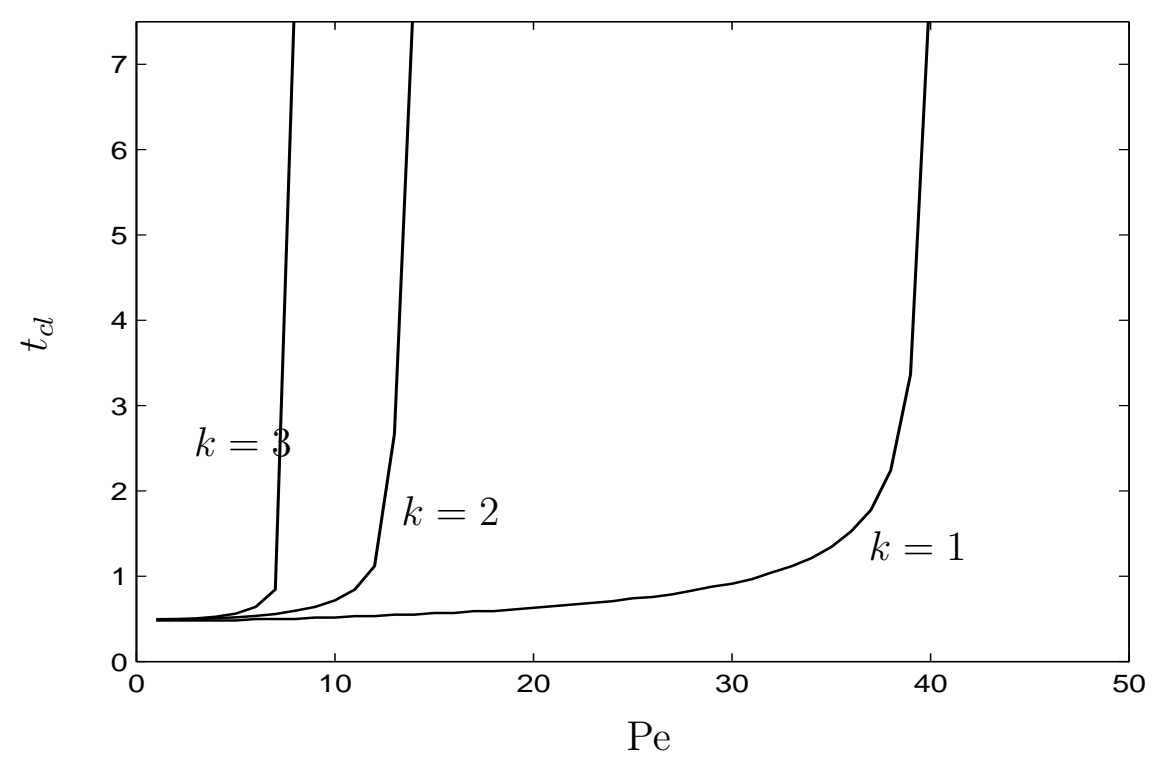

FiguRE 8. Cartesian channel closure time as a function of the Péclet number for $k=1,2,3$.

Figure 8 displays the closure time against Péclet number for 3 values of $k$. An important feature of this figure is that for a large range of Péclet numbers the closure time is approximately constant. For example, with $k=1$ we see that for $P e \leq 10$ the closure time is fixed at $t_{c l}=0.5$. Examination of Figs. 2 and 5 allows us to explain this result. For small Péclet numbers the ice shape is approximately flat along most of the channel and so when closure occurs there is ice almost everywhere. As $P e$ increases the ice shape becomes more curved. When closure occurs, at $x=1$ there may still be a large ice free region. However, the closure shape is irrelevant to the time, once the channel closes the flow stops and the trapped fluid must then also freeze. When $k=1$ the value $P e=10$ may be seen as marking a transition. For $P e>10$ the water flow becomes more important and the closure time slowly increases until near $P e=40$ where there is a rapid increase to infinity. For a given fluid $k$ effectively specifies the wall temperature and so for a wall temperature that results in $k=1$ we see that the flow rate can be increased until around $P e=40$, beyond this the channel will never close. Although we cannot compare directly with the results of Chen et al [5] (their cooling device is outside a polycarbonate structure that encloses rectangular channels) the behaviour shown in Fig. 8 is the same as the experimental and numerical results shown in Fig. 4 of their paper. They find that for flow rates below $10 \mu \mathrm{l} / \mathrm{min}$ the closure time remains at approximately $6 \mathrm{~s}$. As the flow rate increases above $40 \mu \mathrm{l} / \mathrm{min}$ the closure time increases rapidly. A different form is shown in Gui et al [9] in that their closure time always increases with the 
flow rate. This may be because they work with intermediate flow rates or be a result of their different setup that involves multiple channels and a pre-cooling section. Increasing $k$ is equivalent to increasing the wall temperature and so it becomes more difficult to close the channel, hence the critical Péclet number decreases as $k$ increases. For $k=2$ we see that the channel will never close for $P e$ slightly above 14 . Note, that both $k$ and the time-scale are proportional to $1 /\left(T_{f}-T_{w}\right)$ so although the non-dimensional closure time tends to 0.5 for small $P e$ the dimensional time will vary with $k$. If we allow $T_{w} \rightarrow T_{f}$ then it is clear that the dimensional closure time increases rapidly. From these observations we can provide a value for the dimensional closing time, $\bar{t}_{d}$, for low Péclet numbers

$$
\bar{t}_{c l}=\tau t_{c l}=0.5\left[\frac{\rho_{s} L_{f} R^{2}}{k_{s}\left(T_{f}-T_{w}\right)}\right] .
$$

Taking the parameter values of Table 1 we find that for a channel of height $2 h=160 \mu \mathrm{m}$ this leads to $\bar{t}_{c l} \approx 1.72 /\left(T_{f}-T_{w}\right)$ s. When $2 h=400 \mu \mathrm{m}, \bar{t}_{c l} \approx$ $10.7 /\left(T_{f}-T_{w}\right)$ s.

If we consider the evolution of ice in a circular pipe then the shapes are similar to those shown for the Cartesian problem. Noting that for sufficiently small $P e$ and large $x$ then the governing equation is well approximated by Eq. (57), setting $h=0$ in Eq. (58) determines $t_{c l}=0.25$. In this case the dimensional closure time is given by Eq. (72), but the factor 0.5 is replaced with 0.25 . The result is demonstrated clearly in Fig. 9 where we show the pipe closure time against the Péclet number. The main difference between the pipe and Cartesian cases is that the flow rate has to be much higher in the pipe before closure is prevented. For example with $k=1$ closure occurs up to $P e \approx 236$. Presumably this occurs because the pipe is being cooled all around the sides and so feels the wall temperature over a larger region, whereas the two-dimensional channel only has cooling at the top and bottom. The effect on the evolution when $P e$ changes from 236 to 237 is shown in Figs. 10 and 11. In Fig. 10 the ice front slowly evolves until just after $t=10$ closure occurs at the end point $x=1$. The governing equations for this problem are non-linear and often in non-linear problems a small change in parameter values can have a large effect on the solution behaviour. This is clear from Fig. 11 where simply increasing the Péclet number to 237 stops the pipe from ever closing. In the figure we stop the calculation at $t=40$, when the minimum ice height is $h \approx 0.5$. However, if we decrease the value of $k$ to 0.8 (make the wall cooler) and keep $P e=237$ then closure occurs around $t=1$.

We define the critical Péclet number, $P e_{c}$, as the value above which closure never occurs. In Fig. 12 we present results for the reciprocal critical Péclet number against $k$ for both Cartesian and pipe flow. The limit $k \rightarrow 0$ represents either a very cold wall or a liquid entering the pipe very close to the phase change temperature. In either case it is very easy to solidify the liquid. To prevent closure therefore requires a very high flow rate, $P e_{c r} \rightarrow \infty$. Obviously it would 


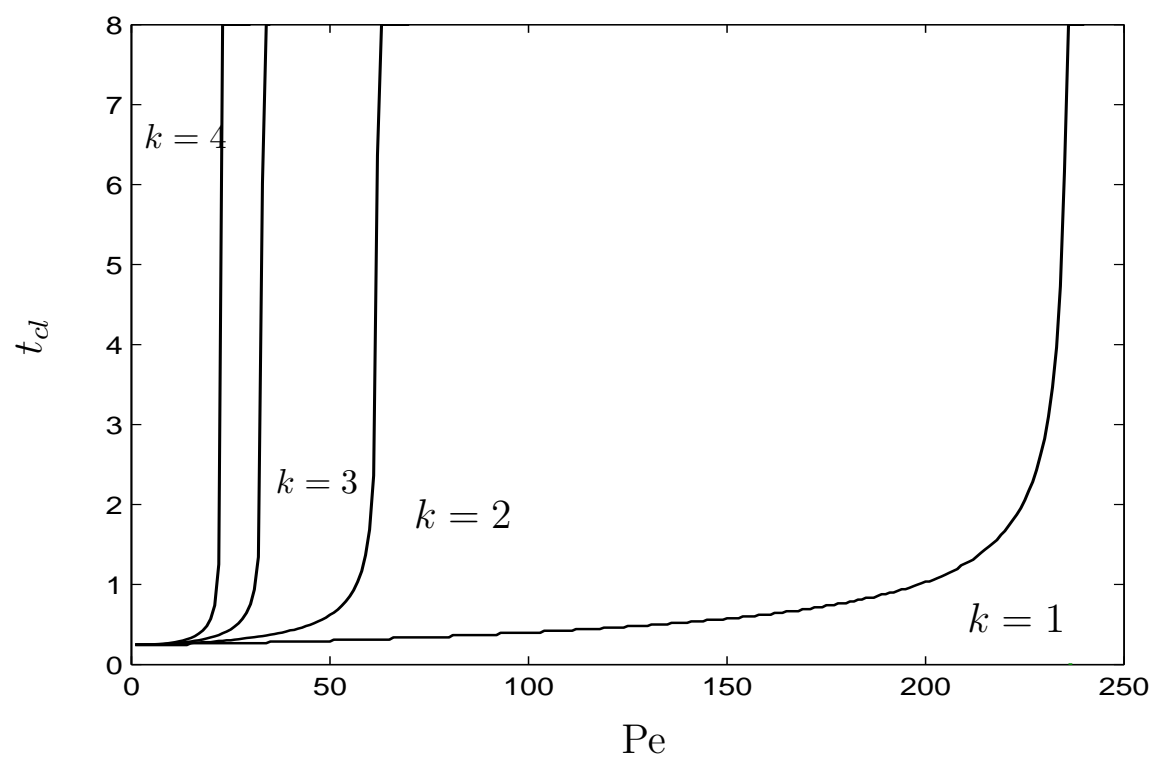

FiguRE 9. Plot of pipe channel closure times (non-dimensional) against Péclet numbers for the pipe.

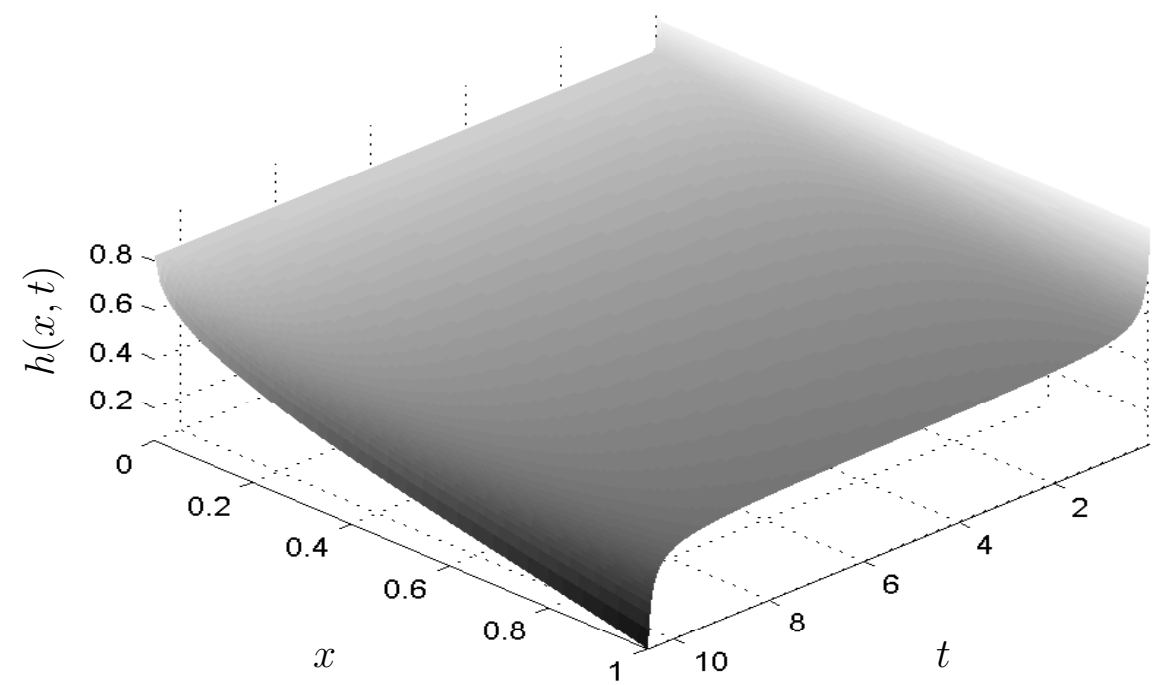

Figure 10. The evolution of the ice front $h(x, t)$ when $P e=236$ and $k=1$ 


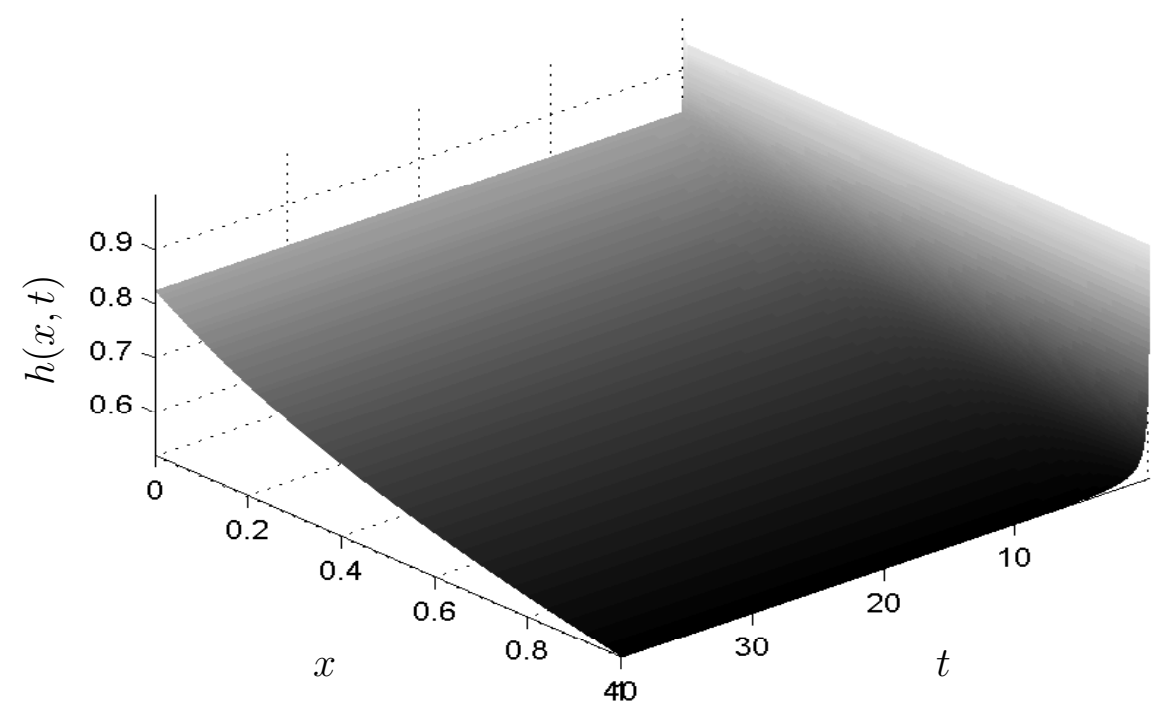

Figure 11. The evolution of the ice front $h(x, t)$ when $P e=237$ and $k=1$.

be difficult to obtain numerical results in these limits but the above argument clearly indicates that the curves shown on the figure must tend to the origin. The approximately straight line results shown on the graph indicate a simple relation of the form

$$
\frac{1}{P e_{c}}=A k-B
$$

Using a least-squares fit we find $(A, B)=(0.0482,0.022),(0.0126,0.0078)$ for the Cartesian and circular channels respectively. These are represented by the dashed lines on Fig. 12. Given that these lines mark the transition from a closing to a non-closing valve we note that the valve operation should always be such that for a given $k$ the flow satisfies $P e \gg 1 /(A k-B)$, i.e. one should operate well above the appropriate line on the figure.

\section{Conclusion}

We have developed a mathematical model for solidification in two-dimensional Cartesian and radially symmetric channels subject to a fixed temperature boundary condition. Unlike previous analyses of solidification in microchannels we fully coupled the flow and freezing problems. Non-dimensionalisation then allowed us to identify the dominant terms and reduce the problem to solving a single integrodifferential equation for the solid thickness.

The solutions demonstrated that for low flow rates the solid is approximately flat along the channel and when closure occurs the channel is already almost 


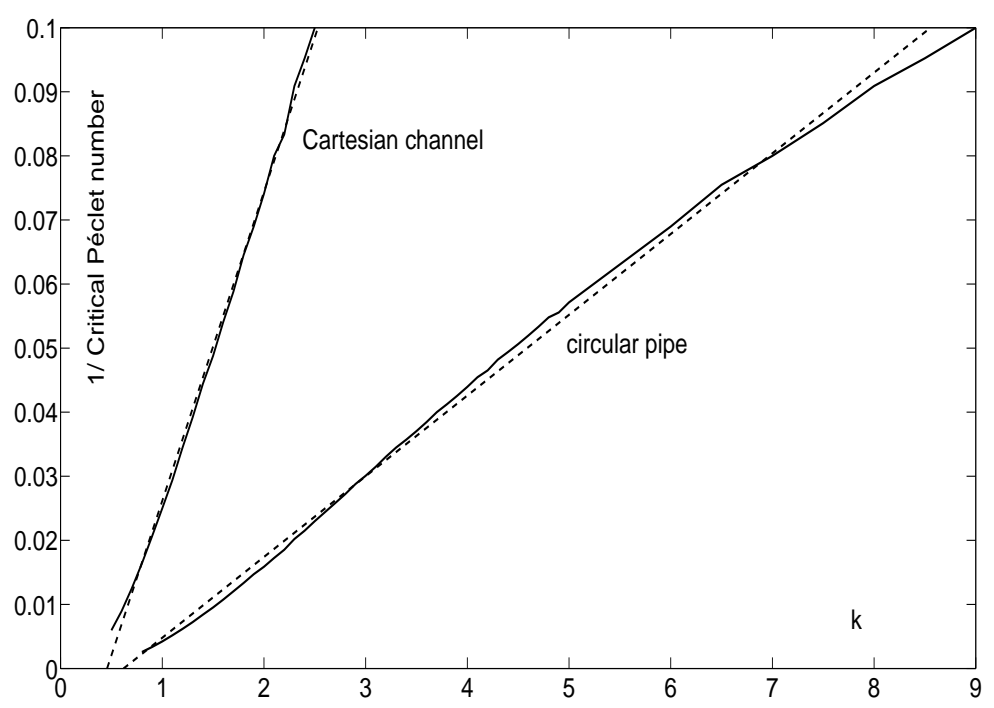

Figure 12. Plot of the inverse critical Péclet numbers against $k$ for both Cartesian and circular channel cases. Solid line shows numerical results, the dashed line is the linear approximation.

full of the solid. For higher flow rates the solid shape is more curved and when closure occurs there is still a large liquid region. This observation explains the fact that for a wide range of flow rates (or Péclet number) we found that the nondimensional closure time was approximately constant and thus may be accurately predicted through a simple equation. Practically this equation tells us that $t_{c l} \propto$ $R^{2} /\left(T_{f}-T_{w}\right)$, that is, provided the flow rate is not too high the closure time depends primarily on the pipe radius (or height) and the difference between the solidification and wall temperatures. However, beyond a certain value the closure time increases with flow rate. Beyond a critical $P e$ the valve will no longer close. The value of the critical $P e$ was shown to vary approximately with the reciprocal of $k, P e_{c} \propto 1 / k$ and this observation then allowed us to define a simple operating criteria.

\section{ACKNOWLEDGEMENTS}

TM gratefully acknowledges the support of this research through the Marie Curie International Reintegration Grant Industrial applications of moving boundary problems, grant no. FP7 - 256417 and partial support by the project 2009-SGR-345 from AGAUR-Generalitat de Catalunya. JL acknowledges postdoctoral funding through the Centre de Recerca de Matemàtica. 


\title{
REFERENCES
}

[1] Abramowitz M, Stegun IA (1972) Handbook of Mathematical Functions with Formulas, Graphs, and Mathematical Tables, 9th edn. Dover Inc. New York

[2] Bevan CD, Mutton IM (1995) Freeze-thaw flow management: a novel concept for high performance liquid chromatography, capillary electrophoresis, electrochromatography and associated techniques. J. Chromatogr A 697:541-548

[3] Bevan CD, Mutton IM (1995) Use of Freeze-Thaw Flow Controlling and Switching Tubes. Anal. Chem. 67:1470-1473

[4] Carslaw HS, Jaeger JC (1959) Conduction of heat in solids, 2nd edn. Clarendon Press, Oxford

[5] Chen Z, Wang J, Qian S, Bau HH (2005) Thermally-actuated, phase change flow control for microfluidic systems. Lab Chip 5:1277-1285

[6] Crank J (1984) Free and Moving Boundary Problems. Oxford University Press, New York

[7] Felton MJ (2003) The new generation of microvalves. Anal. Chem. 75(19):429 A - $432 \mathrm{~A}$.

[8] Gui L, Liu J (2004) Ice valve for a mini/micro flow channel. J. Micromech. Microeng. 14:242246

[9] Gui L, Yu BY, Ren CL, Huissoon JP (2010) Microfluidic phase change valve with a twolevel cooling/heating system. Microfluid. Nanofluid. doi:10.1007/s10404-010-0683-3

[10] He Y, Zhang YH, Yeung ES (2001) Capillary-based fully integrated and automated system for nanoliter polymerase chain reaction analysis directly from cheek cells. J Chromatogr A 924:271-284

[11] Lister JR, Dellar PJ (1996) Solidification of pressure-driven flow in a finite rigid channel with application to volcanic eruptions. J. Fluid Mech. 323:267-283

[12] Mitchell SL, Myers TG (2010) Application of Standard and Refined Heat Balance Integral Methods to One-Dimensional Stefan Problems SIAM Rev. 52 doi:10.1137/080733036

[13] Myers TG, Charpin JPF, Thompson CP (2002) Slowly accreting ice due to supercooled water impacting on a cold surface. Phys. Fluids 14:240

[14] Myers TG, Fowkes ND, Ballim Y (2009) Modeling the Cooling of Concrete by Piped Water J. Eng. Mech. doi:10.1061/(ASCE)EM.1943-7889.0000046

[15] Pal R, Yang M, Johnson BN, Burke DT, Burns MA (2004) Phase change microvalve for integrated devices. Anal. Chem. 76:3740-3748

[16] Weigand B, Braun J, Neumann SO, Rinck KJ (1997) Freezing in forced convection flows inside ducts: A review. Heat Mass Transf. 32:341-351

[17] Yang B, Lin Q (2009) A Latchable Phase-Change Microvalve With Integrated Heaters. J. Microelectromech. Syst. 18:860-867

[18] Yortsos YC, Athanassios KS (2001) Phase change in porous media. Curr. Opin. Colloid Interface Sci. 6:208-216

[19] Zhang C, Xing D, Li Y (2007) Micropumps, microvalves, and micromixers within PCR microfluidic chips: Advances and trends. Biotechnol. Adv. 25:483-514

Centre de Recerca Matemàtica

\author{
Campus de Bellaterra \\ EDIFICI C \\ 08193 Bellaterra, Barcelona \\ SPAIN \\ TEL.: +34-935812951 \\ FAX: +34-935812202 \\ E-mail address: tmyers@crm.cat
}

